\title{
Observation and analysis of interferences on undulator coherent radiation stored in an optical cavity
}

\author{
R. Prazeres, F. Glotin, and J.-M. Ortega \\ CLIO/LCP, bâtiment 201 P.2, Université Paris-Sud, CNRS, Université Paris-Saclay, 91405 Orsay, France
}

(Received 26 June 2018; published 14 November 2018)

\begin{abstract}
We measured the interferences of the coherent radiation, produced by a train of electron bunches in an undulator and stored in an optical cavity. The device used here is a free-electron laser, whose optical cavity stores the radiation pulse for a multipass amplification process. The amplification process is avoided by detuning the optical cavity. This is done by increasing the cavity length, producing a desynchronism between the radiation pulses and electron bunches. Then, the output intensity is dependent on the cavity lengthening and exhibits periodical interferences. An analytical analysis makes a description of this effect. In practice, it allows a direct and simple measurement of the position of the "zero detuning," corresponding to the perfect synchronism of the light pulse and electron bunch in the free-electron laser process.
\end{abstract}

DOI: $10.1103 /$ PhysRevAccelBeams.21.110703

\section{INTRODUCTION}

The CLIO facility is based on an infrared free-electron laser (FEL), provided to a large community of users [1-3]. It uses an optical cavity with two mirrors, separated by a distance $L_{\text {cav }} \approx 4.8 \mathrm{~m}$ and adjusted to ensure synchronization between the laser and electron micropulses. Both of them are 1-2 $\mathrm{mm}$ long (about 5 ps duration), and the tuning curve (Fig. 1) of the laser is even smaller, typically a few wavelengths long. The time structure of the CLIO accelerator is made of macropulses with a repetition rate of $25 \mathrm{~Hz}$ and a time duration of about $10 \mu \mathrm{s}$. These include 600 micropulses separated by $16 \mathrm{~ns}$, corresponding to $2.4 \mathrm{~m}$, which is half of the cavity round-trip time; as a consequence, there are always two light pulses inside the optical cavity.

Two effects must be taken into account for the synchronism condition. These effects occur in the undulator section: slippage and lethargy. The first one, slippage, is due to the difference of speed between the light and the electron bunch in the undulator. The light pulse is moving forward to the front part of electron bunch, by a distance $N \lambda_{R}$, where $N$ is the number of undulator periods and $\lambda_{R}$ is the resonance wavelength. The lethargy [4] effect is due to the FEL gain on the electron bunch, which amplifies principally the back part of the light pulse because of slippage. This effect makes a reduction of the group velocity of the light pulse inside the undulator. Therefore, the cavity

Published by the American Physical Society under the terms of the Creative Commons Attribution 4.0 International license. Further distribution of this work must maintain attribution to the author(s) and the published article's title, journal citation, and DOI. length has to be shortened in order to compensate for the slippage and the reduction of speed of the laser pulse. This results in a typical "tuning curve" of the laser power, as a function of the cavity length, which is shown in Fig. 1.

\section{MEASUREMENT OF RADIATION AT VARIOUS CAVITY LENGTHS}

The zero point corresponds to the "perfect synchronism." The laser power fades out for positive values at a short distance from this point (a few tens of micrometers) because of the previously mentioned effects leading to desynchronization. Nevertheless, using a sensitive detector (nitrogen-cooled $\mathrm{HgCdTe}$ ), a weak signal can be observed in this area despite the disappearance of the laser. This signal is due to a mixing of two effects: (i) laser amplification far below the saturation level [5] and (ii) coherent

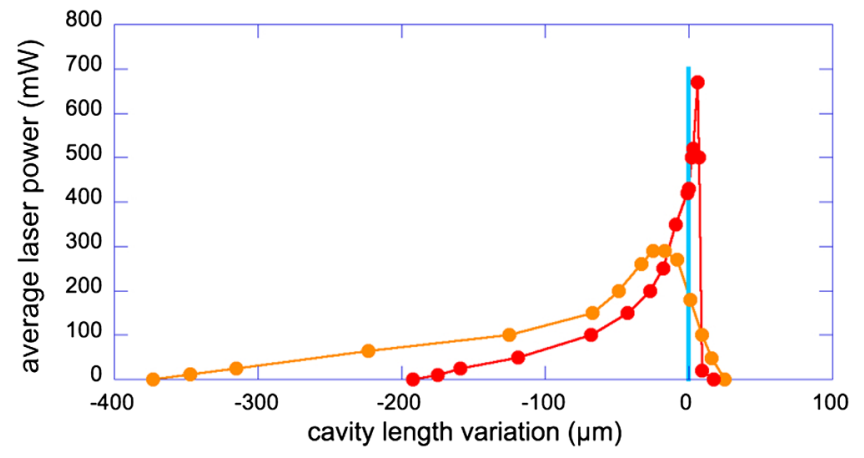

FIG. 1. Variation of laser power of CLIO vs cavity length tuning, at laser wavelength $\lambda=34.6 \mu \mathrm{m}$ for $23 \mathrm{MeV}$ electron beam energy. Two curves are displayed for a different tuning of the electron beam. The blue line at the origin is the perfect synchronism. 


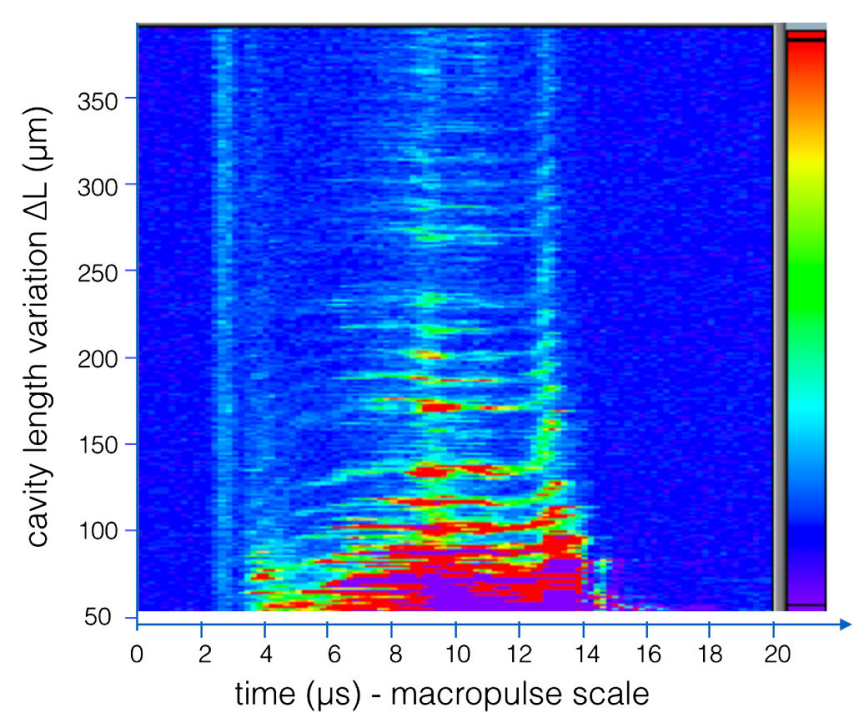

FIG. 2. Intensity of undulator radiation vs time, at the macropulse timescale, for various positions of cavity length (no laser occurs here because out of range), at radiation wavelength $\lambda=31.7 \mu \mathrm{m}$.

emission [6]. The contribution of the laser amplification is stronger when the cavity length is close to the area of perfect synchronism, and it is exclusively dominant at saturation. The coherent emission is strongly dependent on the shape of the microbunches of electrons [7]. It is intrinsically noisy and difficult to control in accelerators. It occurs either when the electron pulse is shorter than the emitted wavelength or when it exhibit substructures of this order of magnitude, which is the case at CLIO [8]. Unfortunately, it is not possible to measure the electron bunch distribution with a resolution much better than $1 \mathrm{ps}$.

Measuring the detector signal as a function of the cavity length, still out from the laser operation set point, allowed us to observe an interference pattern as shown in Fig. 2. This figure shows the light intensity (rainbow scale), the horizontal axis is the timescale, and the vertical one is the cavity length. The full range of the time axis is $20 \mu \mathrm{s}$ and shows the macropulse, which is approximately $10 \mu \mathrm{s}$ duration. The purple area at the bottom is due to the contribution of the laser amplification, because the cavity length is in the vicinity of the laser operation point, and the multipass amplification is sufficient to saturate the detector. When we move the cavity length toward shortened values, i.e., at the up-side part of the red area, no or little amplification occurs, but a remaining signal is still observed. This signal exhibits a periodical pattern. The periodicity is about $15.8 \mu \mathrm{m}$, which corresponds to half of the radiation wavelength $31.7 \mu \mathrm{m}$. Figure 3 represents a $Y$ cut of Fig. 2, and it exhibits clearly the periodical peaks according to the cavity length variation. These interferences are decreasing at a large cavity detuning. In addition, the background of the curve at $\Delta L<100 \mu \mathrm{m}$ corresponds to the remaining contribution of laser amplification close to

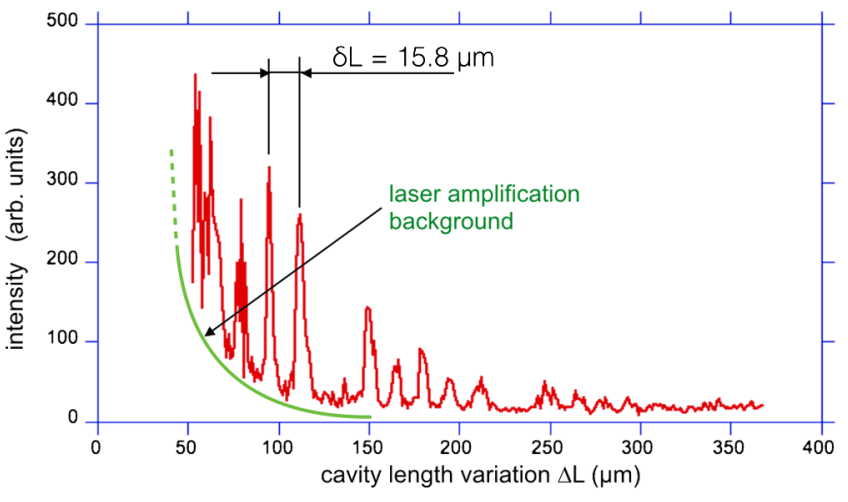

FIG. 3. $Y$ cut of the plot in Fig. 2, showing the radiation intensity vs cavity length variation.

the operation point. The interferences' effect is described in Sec. IV.

Figure 2 shows other phenomena, too. The lacunary appearance of the amplitude distribution is due to the strong variations of coherent emission along the macropulse, showing, for example, a larger peak at $t=9-10 \mu \mathrm{s}$. On the other hand, the sinuous appearance of the periodical curves is due to variations of the centroid energy $\gamma m c^{2}$ of the electron beam along the macropulse. This creates a variation of the laser wavelength according to the undulator resonance condition $\lambda_{R}=\lambda_{o} / 2 \gamma^{2}\left(1+K^{2} / 2\right)$, where $\lambda_{o}$ and $K$ are undulator parameters. As an example, Fig. 4 shows a measurement, made on the CLIO FEL, of such an effect on the laser spectrum vs time. The timescale shows the macropulse, with an arbitrary origin. The lasing starts with the spontaneous emission of a broad spectrum at $t=5 \mu \mathrm{s}$ and reaches saturation with narrowing the spectrum. The centroid wavelength shows an upward drift toward the end

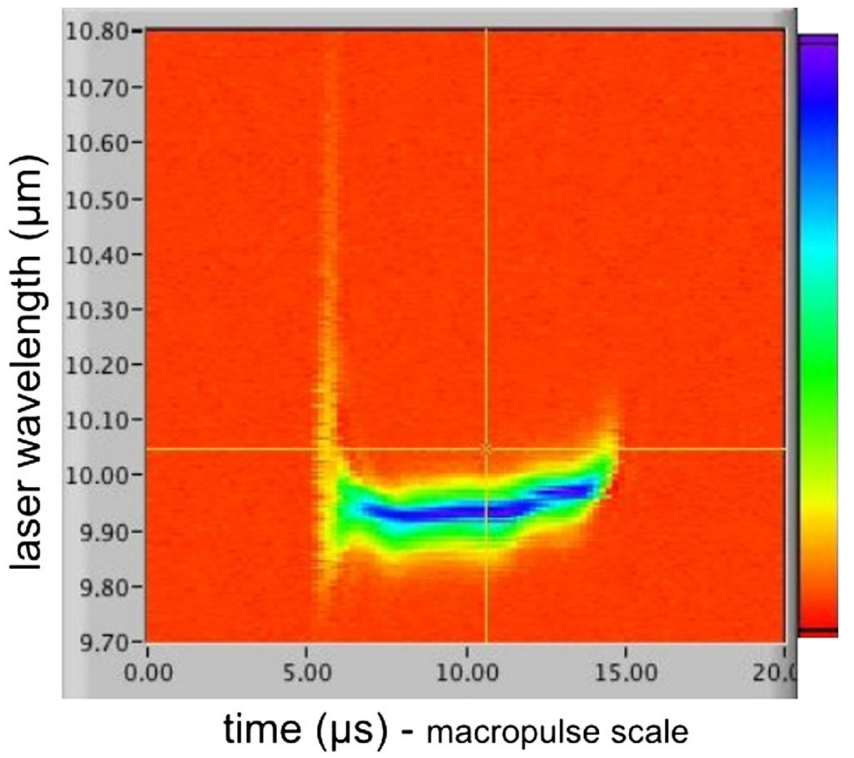

FIG. 4. Power spectrum of the CLIO laser vs time, at the macropulse timescale. 
of the macropulse, due to a decreasing of the electron beam energy. We can see the same behavior in Fig. 2, which exhibits a shift of the interference pattern along the macropulse, corresponding to a shift of the centroid wavelength $\lambda_{R}$.

\section{MEASUREMENT OF RADIATION FOR VARIOUS UNDULATOR GAPS}

The same kind of measurement was done, keeping the cavity length constant and varying the undulator gap. The cavity length $L_{\text {cav }}$ is adjusted toward a long cavity in order to set the FEL laser power to zero. Figure 5 shows the signal vs the time and undulator gap. The periodical interferences are clearly visible according to the undulator gap variation. In addition, it is interesting to note that the interference oscillations are observed here only within a short time duration of about $2 \mu \mathrm{s}$, which represents a small part of the full macropulse of $10 \mu \mathrm{s}$ long. As mentioned above, this is due to the fact that the features of the micropulse are evolving along the macropulse.

Figure 6 represents a $Y$ cut of Fig. 5, and the horizontal axis was calculated into wave numbers $\left(\mathrm{cm}^{-1}\right)$ from the undulator gap values (millimeters). The period of this curve allows us to determine the absolute value of the cavity detuning. Indeed, the perfect synchronism, or "zero detuning," occurs when, after one cavity round trip, the wave front of light comes back at the same position upon the next electron bunch. If the cavity length is increased by $\Delta L$, then the delay of wave front is $2 \Delta L$ after one cavity round trip. Therefore, the laser wave overlaps coherently at each cycle if we have $2 \Delta L=n \lambda$, where $n$ is the interference order. When the cavity length is kept constant, and the wavelength is adjusted, then the interference condition leads to a series

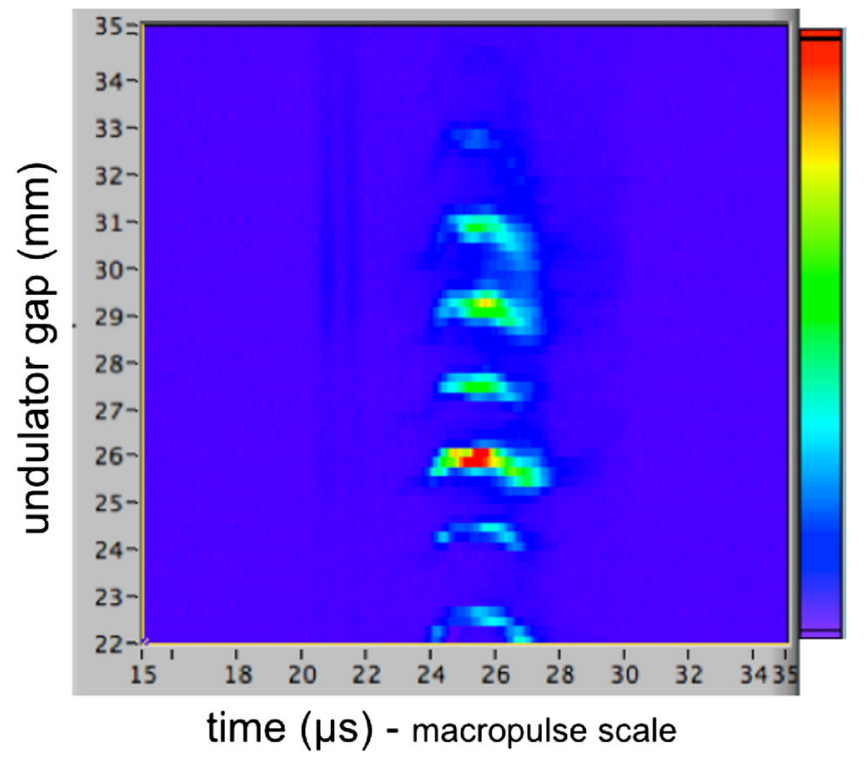

FIG. 5. Intensity of radiation vs time, at the macropulse timescale, for various undulator gaps. of matching wavelengths $\lambda_{n}=2 \Delta L / n$. The relevant wave number series is

$$
\sigma_{n}=n / 2 \Delta L
$$

with $\Delta L$ in centimeters. The periodicity is

$$
\Delta \sigma=\sigma_{n+1}-\sigma_{n}=1 / 2 \Delta L
$$

This gives the position $L_{o}$ of the zero detuning point:

$$
L_{o}=L_{\mathrm{cav}}-\Delta L=L_{\mathrm{cav}}-1 / 2 \Delta \sigma
$$

where $\Delta L=1 / 2 \Delta \sigma$ is the distance between the cavity length $L_{\mathrm{cav}}$ and the zero detuning point $L_{o}$. The expression (3) gives the position $L_{o}$ of the zero detuning point, i.e., the distance between the position of the output mirror during the measurement and the zero detuning point on axis. This method does not give the absolute value of the cavity length (the distance between mirrors of the cavity).

In practice, in the case of CLIO, the variation of the cavity length $L_{\text {cav }}$ is measured by an optical ruler, which gives a reading value $Z$. This ruler is attached to the output mirror of the optical cavity, and it is mounted into the reverse sense: A shortened cavity gives a positive value $Z>0$ on the ruler. The zero position of the ruler $(Z=0)$ corresponds to an arbitrary length $L_{R}$ of the cavity. Therefore, the real cavity length is $L_{\text {cav }}=L_{R}-Z$. See the summary of notations at the end of this paper. Now, the position corresponding to the measurement of Figs. 5 and 6 is $Z=444 \mu \mathrm{m}$ given by the ruler. Therefore, the relevant position $Z_{o}$ of the optical ruler, which corresponds to the zero detuning, is $Z_{o}=Z+\Delta L=Z+1 / 2 \Delta \sigma$ : The sign is inverted here as compared to Eq. (3) because of the reverse sense of the ruler on CLIO. The measurement of Figs. 5 and 6 shows a periodicity $\Delta \sigma=42.5 \mathrm{~cm}^{-1}$. From Eq. (2), we obtain the distance $\Delta L=118 \mu \mathrm{m}$ between the actual position and the zero detuning. Therefore, the zero detuning position on the optical ruler is $Z_{o}=562 \mu \mathrm{m}$ (corresponding indeed to a shorter cavity length). Figure 7 shows the result of various measurements, at different cavity

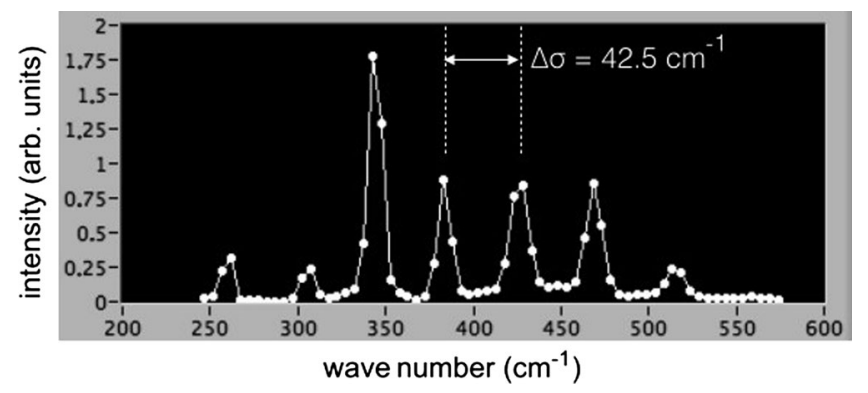

FIG. 6. $\quad Y$ cut of Fig. 5, showing the intensity of radiation vs centroid wavelength of the radiation. 


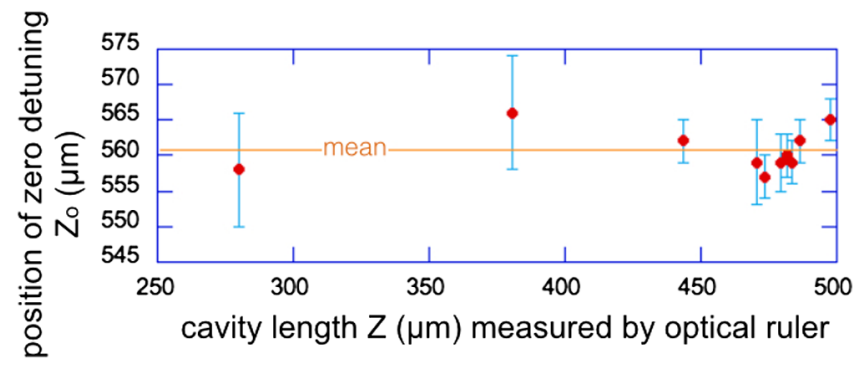

FIG. 7. Series of measurements of the position of zero detuning of cavity length.

lengths: The average position of zero detuning is $\left\langle Z_{o}\right\rangle=$ $561 \mu \mathrm{m}$ with a standard deviation error of $3 \mu \mathrm{m}$. As a summary, this is a very simple and accurate method to measure the zero detuning of the optical cavity of a freeelectron laser. It requires only a measurement of power vs the undulator gap, using a sensitive detector at the output of the cavity. Note that the origin of the abscissa axis on the curve in Figs. 1 and 3 was obtained by this method: The cavity length $\Delta L=0$ corresponds to zero detuning.

This method of measurement of the zero detuning would be useful for studies of the FEL behavior against the cavity length detuning, such as limit-cycle oscillations and supermode operation in the case of short electron bunches [9]; lasing dynamics at zero detuning of the optical cavity, leading to sustained saturation and high-efficiency lasing [10]; generation of stable comb structures in the longitudinal distribution of the radiation pulse, in the case of a deep saturated regime, and at zero detuning of the cavity [11]; and generate stabilized few-cycle optical pulses on the FEL whose cavity length is perfectly synchronized. These few-cycle pulse are stabilized by external laser pulses, overlapping the electron bunch head. This allows one to freeze the amplitude and phase of the shot noise in the leading part of the FEL pulse. The aim is to drive a photon source based on high harmonic generation (HHG) [12].

Note that, in the CLIO case, the accelerator design, based on a double-bend achromat, is not strictly isochronous and keeps a residual anisochronicity of 1 ps per percent of energy. Therefore, an energy slope of electrons during the macropulse, as shown in Fig. 4, produces a small time shift on the successive electron bunches, which is equivalent to a detuning of the cavity length synchronization. Such a "dynamic cavity desynchronism" was deliberately used by the Dutch team of FELIX with a different scheme [13]. In principle, this effect adds to the effective cavity detuning due to mirror displacement $\Delta L$ and could affect our measurement of the zero detuning position $\left\langle Z_{o}\right\rangle$. The equivalent cavity length variation is $\delta L=L_{\text {cav }}(1 / \gamma) d \gamma / d t(100 \alpha)$, with the anisochronicity $\alpha=1 \mathrm{ps} / \%$. The wavelength slope in Fig. 4 is about $\Delta \lambda=0.05 \mu \mathrm{m}$ over $10 \mu \mathrm{s}$ macropulse duration, at $\lambda \approx 10 \mu \mathrm{m}$. This represents an energy variation of $d \gamma / \gamma=2.5 \times 10^{-3}$ over $10 \mu \mathrm{s}$ and gives an equivalent cavity length variation of $\delta L=0.12 \mu \mathrm{m}$, which is much smaller than the rms error $(3 \mu \mathrm{m})$ of measurement of the zero detuning position $\left\langle Z_{o}\right\rangle$ (see Fig. 7). Therefore, the anisochronicity on CLIO does not affect the measurements.

\section{THEORETICAL ANALYSIS}

This effect of interferences is due to the coherent overlap of the recycled and delayed light pulse inside the optical cavity. It requires a production of coherent emission [6] by the electron bunch in the undulator, and this occurs when the bunch distribution contains sharp edges that are creating high-frequency components in the Fourier transform spectrum. The spontaneous emission train [14] emitted by a single electron into an undulator of $N_{u}$ periods can be written

$$
a(t)=\Pi(t / \delta t) \sin \left(2 \pi \nu_{o} t\right),
$$

where $\delta t=N_{u} / \nu_{o}$ is the pulse duration and $\nu_{o}=c / \lambda$ is the radiation frequency corresponding to the undulator resonance. The function $\Pi(x)$ is 1 when $0 \leq x<1$ and zero outside. The relevant amplitude spectrum is

$$
\widetilde{a}(\nu)=\delta t \operatorname{sinc}\left[\pi\left(\nu-v_{o}\right) \delta t\right] e^{i \phi},
$$

where $\operatorname{sinc}(x)=\sin (x) / x$, and the phase $\varphi=\pi\left(\nu-\nu_{o}\right) \delta t$ is due to the centroid position of the $\Pi(x)$ function on $x=1 / 2$. The amplitude $A(t)$ emitted by a bunch of electrons, of longitudinal density $\rho(t)=d N_{e} / d t$, is

$$
A(t)=\int \rho\left(t^{\prime}\right) a\left(t-t^{\prime}\right) d t^{\prime}=[\rho * a](t) .
$$

The relevant amplitude spectrum is

$$
\widetilde{A}(\mathrm{v})=\widetilde{\rho}(\mathrm{v})\left(\delta t \operatorname{sinc}\left[\pi\left(v-v_{o}\right) \delta t\right]\right) e^{i \phi} .
$$

This represents the amplitude of coherent emission produced by a bunch of electrons. Assuming the spectrum of bunch distribution $\widetilde{\rho}(\nu)$ is rather constant close to $\nu_{o}$, then the coherent emission linewidth of $\widetilde{A}(\nu)$ is equal to the spontaneous one: $\Delta \nu_{\mathrm{SE}}=\nu_{o} / N_{u}$. Figure 8(a) shows an example of an analytical calculation of such a spectrum: The red curve is the amplitude spectrum $\widetilde{\rho}(\nu)$ from a Gaussian electron bunch of $\sigma_{t}=33 \mathrm{fs}$ (or length $\sigma_{z}=10 \mu \mathrm{m}$, which is much shorter than that on CLIO). The product, in Eq. (7), between the red curve and the "sinc" function from a spontaneous emission pulse train (with $N_{u}=40$ periods at $\lambda=30 \mu \mathrm{m}$ ) gives the blue curve in Fig. 8(a), which represents the coherent emission spectrum $\widetilde{A}(\nu)$ emitted by the relevant short electron bunch of $\sigma_{z}=10 \mu \mathrm{m}$ long. The central frequency is $\nu_{o}=c / \lambda=$ $10^{13} \mathrm{~Hz}$ for $\lambda=30 \mu \mathrm{m}$. Coherent emission was measured on CLIO [8] in spite of a much larger electron bunch duration of $\sigma_{t} \approx 10 \mathrm{ps}$. This implies that the electron bunch 

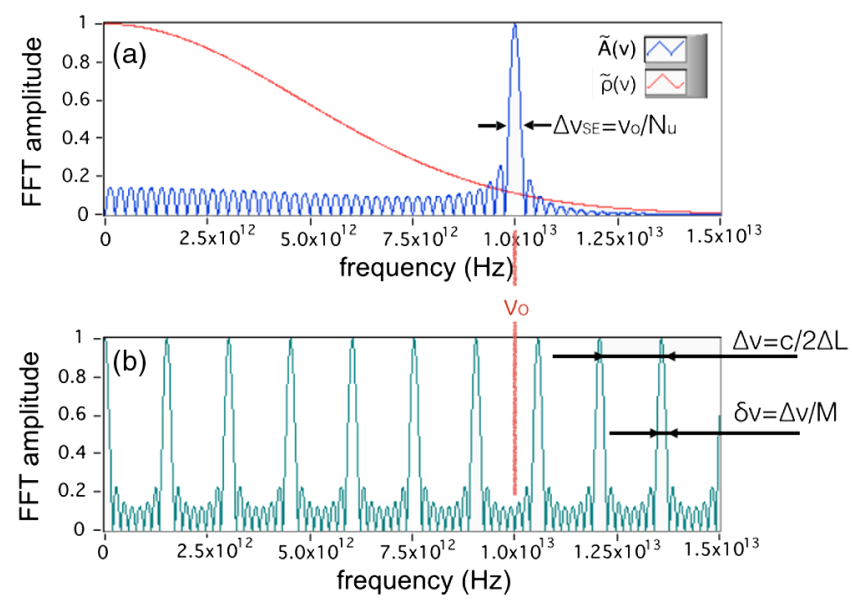

FIG. 8. (a) The blue curve is the analytical calculation of coherent emission $\widetilde{A}(\nu)$ for a single electron bunch with $\sigma_{t}=33 \mathrm{fs}, \lambda=30 \mu \mathrm{m}$, and $N_{u}=40$, peaking at $\nu_{o}=10^{13} \mathrm{~Hz}$, and the red curve is the amplitude spectrum. $\tilde{\rho}(\nu)$ from a Gaussian electron bunch; and (b) is the periodical distribution (Ш $* \operatorname{sinc})$ due to cavity accumulation of light pulses, exhibiting a series of peaks on $\nu_{n}$, with $M=10$ here to allow the clearest view (on CLIO we have $M=300$ ).

distribution $\rho(t)$ is not of the Gaussian type and contains high-frequency components in its spectrum. Indeed, in the Gaussian case, the maximum extension $\sigma_{\nu}=1 / 2 \pi \sigma_{t}$ of the Fourier distribution of $\rho(t)$ is about $10^{10} \mathrm{~Hz}$. Therefore, the distribution does not reach high frequencies such as $\nu_{o}=c / \lambda=10^{13} \mathrm{~Hz}$, and the results of Eq. (7) would be zero. The high-frequency components are due to a sharp edge in the distribution of $\rho(t)$ for the CLIO electron bunches.

Now, the optical cavity makes a recycling of this radiation pulse, with a time delay $\Delta t=2 \Delta L / c$ due to the cavity detuning. At the end of the macropulse, the final amplitude distribution $A_{M}(t)$ inside the cavity is the sum of $M$ delayed pulses, and it becomes

$A_{M}(t)=\sum_{n=0}^{M-1} A(t-n \Delta t)=A(t) *\left[\amalg(t / \Delta t) \Pi\left(t / T_{M}\right)\right]$,

where $\amalg(x)$ is the Dirac comb of the unit period, $\Pi(x)$ is 1 when $0 \leq x<1$ and zero outside, and $T_{M}=M \Delta t$ contains the number on micropulses $M$ involved in the sum. The relevant amplitude spectrum is then

$$
\widetilde{A_{M}}(v)=\widetilde{A}(v)\left\{\amalg(v \Delta t) *\left[T_{M} \operatorname{sinc}\left(\pi \nu T_{M}\right) e^{i \theta}\right]\right\},
$$

where the phase $\theta=\pi \nu T_{M}$ is due to the centroid position of the $\Pi(x)$ function on $x=1 / 2$. As a summary, the spectrum amplitude $\widetilde{A}_{M}(\nu)$ is a product of $\widetilde{A}(\nu)$, centered on frequency $\nu_{o}$, with a series of peaks which are upon the periodical positions $\nu_{n}=n / \Delta t$ of the Dirac comb distribution $\amalg(\nu \Delta t)$. Figure 8 shows an example from an analytical calculation. The blue curve in Fig. 8(a) shows the coherent emission spectrum $\widetilde{A}(v)$ from a single electron bunch. The curve in Fig. 8(b) shows the Dirac comb distribution $\amalg(\nu \Delta t)$ convoluted by the $\operatorname{sinc}\left(\pi \nu T_{M}\right)$ function due to the limited numbers $M$ of pulse series. It corresponds to the analytical description of the recycling of a radiation bunch into the optical cavity, and it is dependent the shift $\Delta L$ between overlapped bunches in the optical cavity. Note that the parameter $M$ represents the number of micropulses which are able to provide coherent emission, and $M$ can be lower than the total number of micropulses produced by the accelerator ( 300 for the case of CLIO). Indeed, this is clearly shown in Fig. 5, as discussed above and where the micropulses are generating coherent emission only within a short time period of $2 \mu \mathrm{s}$, because their features are evolving along the macropulse; and this corresponds to $M=60$.

Now, the positions $\nu_{n}=n / \Delta t$ of the peaks of the Dirac comb are dependent on the cavity detuning length $\Delta L=c \Delta t / 2$. The amplitude of $\widetilde{A}_{M}(\nu)$, which represents the product of the curves from Figs. 8(a) and 8(b) [see Eq. (9)], is then dependent on $\Delta L$. The curve in Fig. 9 shows the energy of coherent emission, i.e., the integration of the square of modulus of $\widetilde{A}_{M}(\nu)$ over the frequency, dependent on the cavity length detuning $\Delta L$. This curve in Fig. 9 shows a periodical behavior with a series of peaks, and each peak corresponds to the coincidence between the undulator frequency $\nu_{o}$ and one of the periodical positions $\nu_{n}=$ $n / \Delta t$ of the Dirac comb distribution $\amalg(\nu \Delta t)$. In other words this occurs when the cavity detuning $\Delta L$ corresponds to the discrete values:

$$
\Delta L(n)=\frac{n \lambda}{2}
$$

where $n$ is the interference order number. This explains the observation of interference oscillations of $\lambda / 2$ periodicity, which are described in Secs. II and III. When the cavity matches these values $\Delta L=\Delta L(n)$, the radiation pulse is adding coherently, due a constructive overlap over the cavity cycles, and the interference gives a larger signal on the detector. For unmatched values of the cavity length, the sum

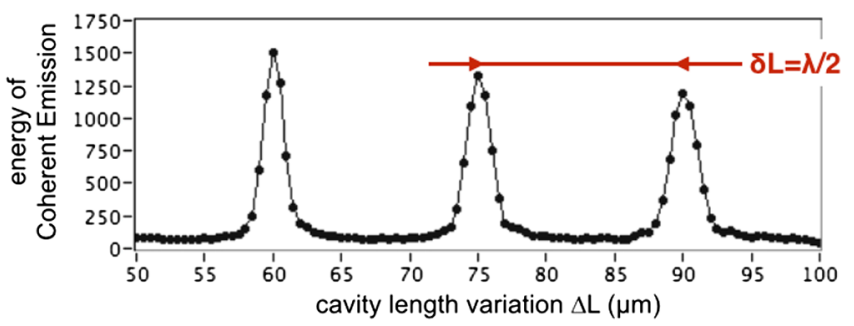

FIG. 9. Analytical calculation of coherent emission: integration over the frequency $\nu$ of the square of modulus of $\tilde{A}_{M}(\nu)$, vs cavity length detuning. 


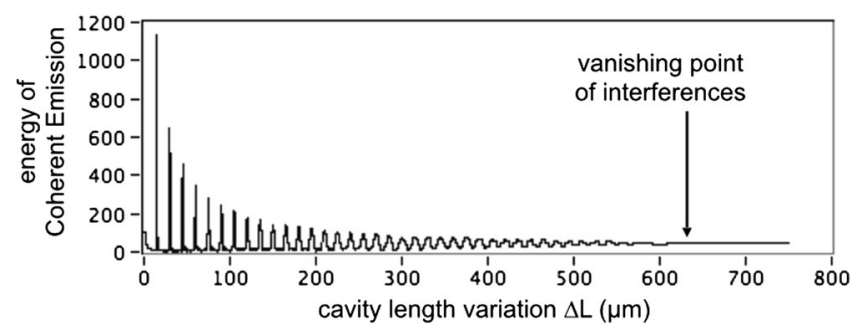

FIG. 10. Coherent emission vs cavity length detuning, within the expanded range, showing vanishing interferences at $\Delta L=$ $630 \mu \mathrm{m}$ when using the parameters of CLIO: $N_{u}=40$ and $\lambda=31.7 \mu \mathrm{m}$.

of the successive contribution becomes incoherent and the output signal vanishes, despite the fact that the various contributions were initially coherent. Also, the overlap of the successive pulse trains decrease as the cavity is lengthened, and their intensity decreases accordingly, as described above.

\section{A. Resolution limit of oscillations}

As we can understand from Fig. 8, the interferences are vanishing when the distance $\Delta \nu$ reduces, i.e., when $\Delta L$ increases. This is shown in Fig. 10, showing an expanded range of Fig. 9. The vanishing point of interferences occurs when the spontaneous emission linewidth $\Delta \nu_{\mathrm{SE}}$ of the function $\widetilde{A}(\nu)$ is equal to the distance $\Delta \nu$ between two peaks of the distribution $\amalg(\nu \Delta t)$. This condition corresponds to the limit

$$
\Delta L_{\text {limit }}=N_{u} \lambda / 2
$$

When making a relation with Eq. (10), we can say that the vanishing point occurs when the interference order $n$ is equal to the number of undulator periods: $n=N_{u}$, or when the distance $c \Delta t$ between micropulses is equal to the coherence length: $c \Delta t=N_{u} \lambda$. Now, when using the experimental parameters which are involved in Fig. 2, with $N_{u}=40$ and $\lambda=31.7 \mu \mathrm{m}$, we obtain an upper limit for interferences of $\Delta L_{\text {limit }}=630 \mu \mathrm{m}$, corresponding to the interference order $n=40$. This calculation corresponds to the experimental curve in Fig. 3. The amplification background in the measurement does not appear here, because the calculation does not take into account the FEL gain. The measurement shows a vanishing point at about $300 \mu \mathrm{m}$, which is shorter than the calculated one at $630 \mu \mathrm{m}$. This discrepancy should be due to noise of the detector, which measures the radiation intensity at the output of the undulator and which does not allow one to discriminate the low level of oscillations from 300 to $600 \mu \mathrm{m}$.

\section{CONCLUSION}

We measured interference effects on the coherent radiation produced by the undulator of a free-electron laser below the laser threshold, using a detuning range of the optical cavity. This effect of interferences appears even if there is no amplification at all. It is due the superposition of the coherent emission pulses produced by the electron bunches in the undulator, which are stored in the optical cavity and are delayed along the round trips by length detuning. The superposition of these pulses is coherent only when the delay is equal to an entire number of wavelengths; otherwise, it tends to add them randomly. This effect is not equivalent to interferences obtained in a Fabry-Perot nor to a Michelson: These passive devices only make a redistribution of the radiation energy, but they do not create energy. In our case, it is a coherent emission process [6] due to a coupling between photons and electrons and producing an additional amount of energy, which occurs periodically as a function of cavity length detuning. It is a very simple and accurate method to measure the position of the zero detuning of the optical cavity of a free-electron laser. It requires only measurements of the power vs the undulator gap, with a sensitive detector at the output of the cavity. This measurement would be useful for studies of the FEL behavior against the cavity length detuning.

\section{A. Summary of notation}

$L_{\text {cav }}$, absolute value of the cavity length (about $4.8 \mathrm{~m}$ on the CLIO FEL); $Z$, value of the cavity length measured by the optical ruler of CLIO; $L_{R}$, cavity length $L_{\text {cav }}$ corresponding to the arbitrary zero point of the optical ruler; $L_{o}$, zero detuning length of the cavity, i.e., when the round-trip time of the cavity at light speed is equal to the time delay between two electron bunches; $Z_{o}$, zero detuning position measured by the optical ruler; $\Delta L=L_{\mathrm{cav}}-L_{o}$ is the difference between the zero detuning $L_{o}$ and the cavity length $L_{\mathrm{cav}}$; $M$, number of micropulses; $N_{u}$, number of undulator periods; $\delta t$, time duration of the individual spontaneous emission pulse train (for one electron); $\Delta t$, time delay due to cavity length detuning; $T_{M}=M \Delta t$, accumulated time delay in the cavity for the $M$ th micropulse; $\sigma=1 / \lambda$ is the wave number in $\mathrm{cm}^{-1} ; \sigma_{z}$, the rms electron bunch length; $\sigma_{t}$, the rms electron bunch time duration; $\nu_{o}$, resonance frequency of the undulator radiation.

[1] R. Prazeres, F. Glotin, and J. M. Ortega, New results of the "CLIO" infrared FEL, Nucl. Instrum. Methods Phys. Res., Sect. A 528, 83 (2004).

[2] R. Prazeres, F. Glotin, J. M. Ortega, C. Rippon, R. Andouart, J. M. Berset, E. Arnaud, and R. Chaput, Study of the "CLIO" FEL properties at long wavelengths, Nucl. Instrum. Methods Phys. Res., Sect. A 445, 204 (2000).

[3] R. Prazeres, F. Glotin, C. Insa, D. A. Jaroszynski, and J. M. Ortega, Two colour operation of a free electron laser and applications in the mid-infrared, Eur. Phys. J. D 3, 87 (1998).

[4] H. Al-Abawi, F. A. Hoff, G. T. Moore, and M. O. Scully, Coherent transients in the free-electron laser: Laser lethargy, and coherence brightening, Opt. Commun. 30, 235 (1979). 
[5] F. Ciocci, G. Dattoli, A. Torre, and A. Renieri, Insertion Devices for Synchrotron Radiation and Free Electron Laser (ENEA INN-FIS, Frascati, Rome, 2000), Chap. 3, p. 81, ISBN 981-02-3832-0.

[6] R. Coïsson, Energy balance in coherent electromagnetic radiation, Eur. J. Phys. 15, 29 (1994).

[7] D. A. Jaroszynski, R. J. Bakker, A. F. G. van der Meer, D. Oepts, and P. W. van Amersfoort, Coherent Startup of an Infrared Free-Electron Laser, Phys. Rev. Lett. 71, 3798 (1993).

[8] J. M. Ortega, R. Prazeres, F. Glotin, and D. A. Jaroszynski, Coherent and self-amplified infrared synchrotron radiation emitted by a 50-MeV electron beam, Phys. Rev. E 57, 1053 (1998).

[9] N. Piovella, P. Chaix, G. Shvets, and D. A. Jaroszynski, Analytical theory of short-pulse free-electron laser oscillators, Phys. Rev. E 52, 5470 (1995).
[10] N. Nishimori, R. Hajima, R. Nagai, and E. J. Minehara, Sustained Saturation in a Free-Electron Laser Oscillator at Perfect Synchronism of an Optical Cavity, Phys. Rev. Lett. 86, 5707 (2001).

[11] P. L. Ottaviani, S. Pagnutti, G. Dattoli, E. Sabia, V. Petrillo, P. J. M. van der Slot, S. Biedron, and S. Milton, Deep saturated free electron laser oscillators and frozen spikes, Nucl. Instrum. Methods Phys. Res., Sect. A 834, 108 (2016).

[12] R. Hajima and R. Nagai, Generating Carrier-EnvelopePhase Stabilized Few-Cycle Pulses from a Free-Electron Laser Oscillator, Phys. Rev. Lett. 119, 204802 (2017).

[13] R. J. Bakker, G. M. H. Knippels, A. F. G. van der Meer, D. Oepts, D. A. Jaroszynski, and P. W. van Amersfoort, Dynamic desynchronization of a free-electron laser resonator, Phys. Rev. E 48, R3256 (1993).

[14] Y. Farge, Emission of photons by undulators, Appl. Opt. 19, 4021 (1980). 